\title{
An Evaluation Method of Assessing the Low Back Muscle Fatigue in Manual Material Handling
}

\author{
A. Komeili ${ }^{1,5 *}, \mathrm{X} . \mathrm{Li}^{2}, \mathrm{M} . \mathrm{Gul}^{3}, \mathrm{~J}$. Lewick$^{4}$, and M. El-Rich ${ }^{3}$ \\ ${ }^{1}$ Postdoc fellow, Department of Civil Engineering, University of Alberta \\ ${ }^{2}$ PhD student, Department of Civil Engineering, University of Alberta \\ ${ }^{3}$ Professor, Department of Civil Engineering, University of Alberta \\ ${ }^{4}$ MBA B.Sc., Glenrose Rehabilitation Hospital, Edmonton, Alberta \\ ${ }^{5}$ Corresponding author's e-mail: akomeili@ualberta.ca
}

\begin{abstract}
Workers in certain industries are exposed to high labor-intensive tasks. Low back pain (LBP) is widespread among construction workers (Hildebrandt, 1995) and is extremely prevalent, with every adult having up to an $85 \%$ chance of experiencing LBP at least once during his or her lifetime. Back-related complaints are more costly than those from any other body part for Alberta work-related claims. Ergonomic principles in addition to the engineering considerations should be included in the design of work stations to minimize the risk of injury for employees. In this study, we assessed the low back muscle fatigue due to lifting tasks. The objective was to investigate the changes in muscle activity and kinematics of the human body caused by fatigue due to repetitive lifting tasks. Three healthy male volunteers with no recent back complications were asked to complete 3 cycles of lifting task at the Syncrude Centre of the Glenrose Rehabilitation Hospital. Each cycle involved lifting a 15lb window frame for 20 times. Selfadhesive reflective markers were attached on the hands, trunk, and legs to measure displacements and rotation of the body parts while performing the task. Electromyography (EMG) sensors were placed on lower back muscles to record their activity. The muscle fatigue was investigated by studying change in EMG spectral parameters such as RMS, mean frequency (MNF), and median frequency (MDF) as well as physical condition of subjects due to repetitive lifting. The power frequency curve shifted to the low frequency when muscle fatigue occurred. As a result, the slope of the RMS, and MNF indicators were successful to describe the fatigue behavior expected.
\end{abstract}

\section{KEYWORDS}

Ergonomics, EMG, Low Back Pain, Muscle Fatigue

\section{INTRODUCTION}

Assessing back muscle fatigue has been the interest of investigators due to its association with low back pain (LBP) and other musculoskeletal disorders (Roy, De Luca, \& Casavant, 1989). With a total of 35,655 back-related lost time claims reported between 2008 and 2013 across all industries, LBP is a major issue among Alberta workers and employers (Atlas \& Deyo, 2001). Additionally, back-related complaints have been more costly than those from the rest of the body for Alberta work-related claims (Atlas \& Deyo, 2001), which led the reduction of LBP to 
become a high priority for Alberta workers and employers. Mechanical load is considered one of the major contributors to LPB onset. One complication of assessing the LBP is quantifying muscle forces and joint loads. Surface electromyogram (sEMG) has been used to collect the electrical potential generated by muscle cells when they are electrically or neurologically activated. The sEMG signals are affected with the extent of muscular involvement during occupational work (Bruno, Bouxsein, \& Anderson, 2015; Marshall, Lovell, Knox, Brennan, \& Siegler, 2015). It was shown that the power-frequency spectrum of a muscle shifts to lower frequency band during the development of fatigue in isometric tasks (Lindström, Kadefors, \& Petersén, 1977; Segizbaeva \& Aleksandrova; Woźniak, Lipski, Lichota, \& Szyszka-Sommerfeld, 2015). Thus analysis of sEMG could be a non-invasive method to predict the development of fatigue before initiating muscle injuries. A review of the literature on fatigue behaviour with sEMG provided different fatigue indicators such as Root-Mean Square (RMS), Average Rectified Value (ARV), Mean Frequency (MNF), and Median Frequency (MDF) (Cao, El Hajj Dib, Antoni, \& Marque, 2007; Georgakis, Stergioulas, \& Giakas, 2003; Kim, Ahad, Ferdjallah, \& Harris, 2007; Mananas, Rojas, Mandrile, \& Chaler, 2005).

The objective of this study is to generate and quantify low back muscle fatigue resulted from repetitive lifting using sEMG data. The sEMG indicators will also allow validate a human body model that will be used in the future to predict the muscle forces and joint loads. The long term objective of this study is to create a proactive ergonomic analysis to reduce the number of workrelated injuries by identifying potentially hazardous tasks.

\section{METHODS}

\section{Subjects:}

Three healthy male subjects with no back pain history (age $30 \pm 3$ years, height $177 \pm 7 \mathrm{~cm}$, and body mass $70 \pm 13 \mathrm{~kg}$ ) were recruited for a lifting task experiment. The test procedure and possible risks involved were explained ahead and a written consent form was signed by each subject.

\section{Data Acquisition:}


From plant observation and physical demand analysis (PDA) in the All Weather Windows Ltd. (AWW), one of the window and door manufactures in Canada, the lifting task was selected to generate low back muscle fatigue. The lifting task simulation was performed in the Syncrude Centre at the Glenrose Rehabilitation Hospital (Edmonton, Alberta). Self-adhesive reflective markers were attached on the specific anatomical locations as illustrated in Error! Reference source not found. in order to record kinematics of the body segments while performing the lifting tasks (Error! Reference source not found.). The reflective markers will be used to drive the human body model in the future studies. An 8-camera Eagle Digital Motion Analysis system (Motion Analysis Corp., Santa Rosa, CA, USA) sampling at $120 \mathrm{~Hz}$ was employed to collect the 3D coordinates of the reflective markers during the test. Two AMTI (advanced Mechanical Technology Inc. MA, USA) force plates sampling at $2400 \mathrm{~Hz}$ were utilized to capture ground reaction forces under participant's feet. The rectus abdominis, multifidus, iliocostalis, and longissimus muscles activities were recorded using 4 pairs of sEMG sensors with sample rate of $2400 \mathrm{~Hz}$. Error! Reference source not found. illustrates the configuration of sEMG system.

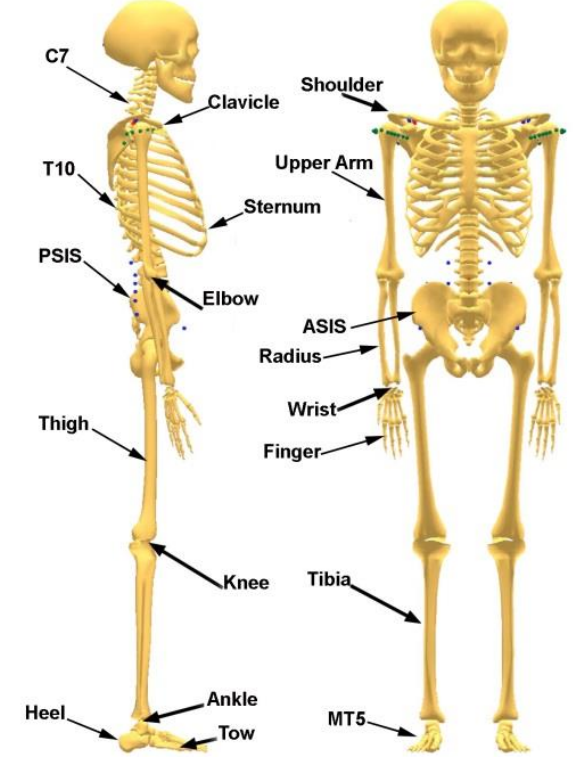

Figure 1- Anatomical positions of 42 reflective markers used to drive the AnyBody manikin model. 


\begin{tabular}{|l|c|c|c|}
\hline & Right & Left & Level \\
\hline Rectus Abdominis & CH1 & CH2 & Umbilicus \\
\hline Multifidus & CH3 & CH4 & L5 \\
\hline Longissimus & CH5 & CH6 & L1 \\
\hline Iliocostalis & CH7 & CH8 & L5 \\
\hline
\end{tabular}

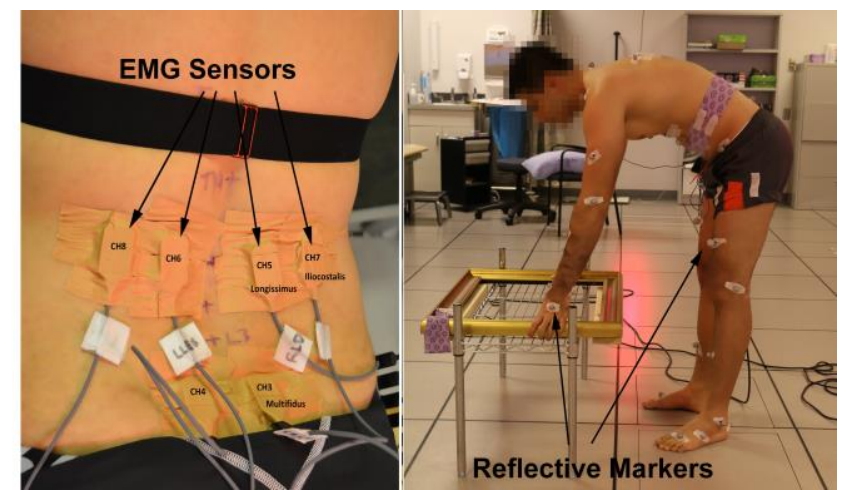

Figure 2- Left: iliocostalis, longissimus, and multifidus sEMG sensors location, Right: MoCap reflective markers are attached to specific anatomical locations while the subject performs the lifting task.

\section{Test Procedure:}

The areas of sensors placement were shaved and washed with alcohol to improve conductance. Repetitive stoop lifting task was considered which involved extension and flexion of the trunk while knees remained straight. One complete cycle of lifting task started with bending forward, without twisting the trunk and bending knees, and continued with lifting a 15lb rectangular window frame from a table, holding it close to the body in upright standing posture, and finished by putting it back to the table as illustrated in Error! Reference source not found. and Figure 3. The weight of the window frame meets the Workplace Safety \& Prevention Services (WSPS) requirements. Three lifting trials of 20 cycles each were performed to generate muscle fatigue. One minute rest between each trial was given to the subject to prevent muscle injury. Two MVC techniques were carried out in different postures. For the trunk extension MVC, subject was strapped in a prone position, with the torso horizontally cantilevered over the end of the bed. The subject then attempted to extend the upper trunk in the sagittal plane(Vera-Garcia, Moreside, \& McGill, 2010). For the rectus abdominis extension, the subject was in a $90^{\circ}$ sit-up posture positioned on a bed with legs strapped. He then attempted to bend backward in the sagittal plane up to horizontal.

\section{sEMG post-processing:}

The sEMG signals were band-pass filtered $(12-1200 \mathrm{~Hz})$ to remove the high frequency noise. The sEMG signals were segmented into epochs, where one epoch started from upright standing posture and ended in the next upright standing posture as shown in Figure 3. Therefore, one full cycle of lifting consisted of two epochs; one for lifting the load, and one for returning the load back to the table. The standard deviations $\sigma(t)$ of epochs were calculated and empirical cut off constant, $\kappa=0.6^{*} \max (\sigma(\mathrm{t}))$, were used to compose a window with the sEMG maximum energy as shown in Figure 3. The sEMG parameters were calculated using a moving Hamming window in which a sliding time window of $1 \mathrm{~s}$ was taken and continuously displaced by $10 \mathrm{~ms}$ along the hatched windows. The aforementioned coefficients were calculated for each time window, then, all coefficients were related to time. The sEMG factors for each channel were normalized using the maximum voluntary contraction (MVC) values obtained before performing the test (Sarmiento et al., 2011) . 


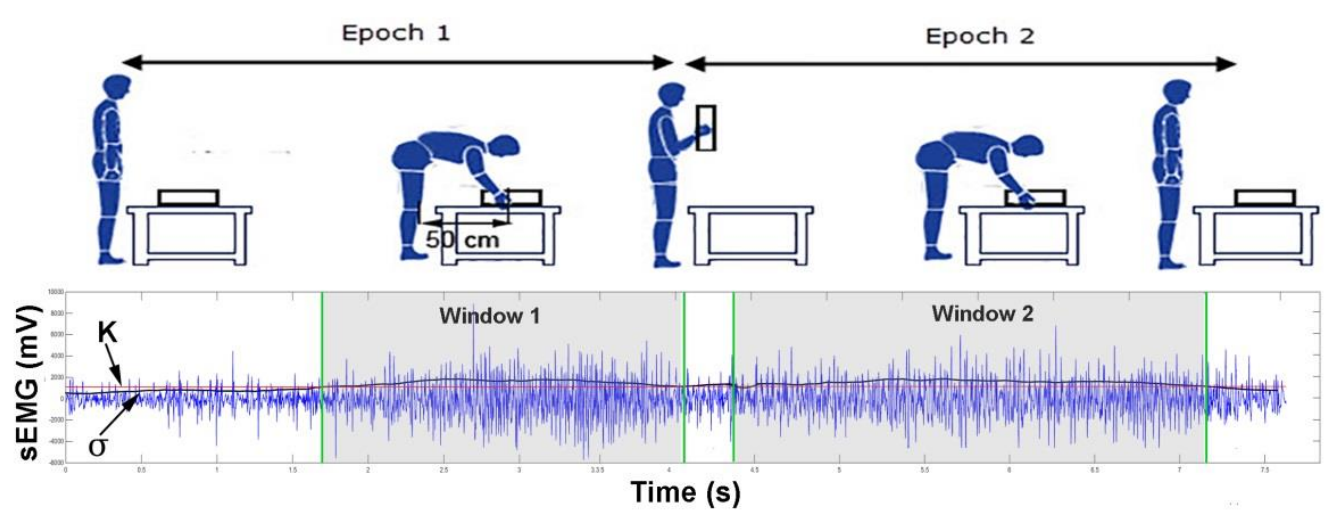

Figure 3- The configuration of a full cycle lifting task with its two epochs. $\kappa$ : cut off line, $\sigma$ : standard deviation, green vertical lines: intersection of $\kappa$ and $\sigma$ curve, hatched area: window with the sEMG maximum energy.

\section{RESULTS}

The physical symptoms such as, respiration rate, heartbeat, and feedback of subjects implied the development of fatigue. For instance, Table 1 shows the mean heartbeat of subjects during the test, which increased constantly as subjects performed the test.

Table 1- The mean heartbeat of subjects in each trial.

\begin{tabular}{|l|ccc|}
\hline & \multicolumn{3}{c|}{ Heartbeat } \\
\cline { 2 - 4 } & Trial 1 & Trial 2 & Trial 3 \\
\hline Subject1 & 97 & 103 & 109 \\
Subject2 & 91 & 96 & 107 \\
Subject3 & 87 & 88 & 103 \\
\hline
\end{tabular}

Investigating the sEMG signals of 8 sensors specified substantial artefact in the data obtained from channels 1 and 2, where the rectus abdominis muscles were located. The low abdominal muscle activity during the test, and considerable layer of fat in the abdominal section were speculated the main reasons affecting the sEMG signals of channels 1 and 2 . Therefore, the study of muscle fatigue development in the abdominal muscles was inevitably avoided. The accuracy of sEMG data collection was validated by placing a pair sEMG sensor longitudinally next to each other on each of multifidus, longissimus, and iliocostalis muscles. Figure 4 compares the RMS for each pair of sEMG channels. Analogous shape of RMS curves determined the validity of data collection. As can readily be seen, the RMS and AVR of sEMG signal did not significantly change intra-trial (within trial), which can partly be attributed to the insufficient load to initiate fatigue in the first trial and to a lesser extent to the synergy of the lower back muscles in handling loads. Although previous studies demonstrated progressive decrease of the MNF and MDF curves for bicep muscle as a function of time inter-and-intra trial (Mannion \& Dolan, 1994), that was not the case in this study. Right column of Figure 4 compares the 
variation of sEMG factors for $\mathrm{CH} 3$ between trial1 and trial3 for subject2. Both MDF and MNF did not represent a consistent behaviour in trial 1 and trail 3, i.e. they fluctuated during the lifting task. One possible explanation is that the low back muscles were not constantly activated during the test, e.g. in standing position, which would severely alter the frequency relationship. Although RMS and AVR magnitude did not change significantly intra-trial, significant decrease of RMS and AVR magnitude was observed inter-trials (between trials). The slight increase of RMS and ARV at the beginning of trial1 stems from combination of many factors such as greater firing rate and activation of more motor units to handle the load (Sparto, Parnianpour, Reinsel, \& Simon, 1997). The decrease of the RMS and ARV within a trial could be the indication of muscle fatigue development (Perry, Easterday, \& Antonelli, 1981; Vøllestad, 1997). Figure 5 illustrates the mean RMS ( $\overline{R M S}$ ) of sEMG in each trial for low back sensors. The lower magnitude of the $\overline{R M S}$ in trial3 for multifidus, longissimus, and iliocostalis muscles with respect to the corresponding values in triall indicated the development of muscle fatigue due to repetitive lifting. As expected the power-frequency curve shifted to lower frequencies in trial3 compare to the trial1 as shown in Figure 6. This behaviour was reported in the literature for isometric contraction of muscles (Kranz, Williams, Cassell, Caddy, \& Silberstein, 1983; Lindström et al., 1977).
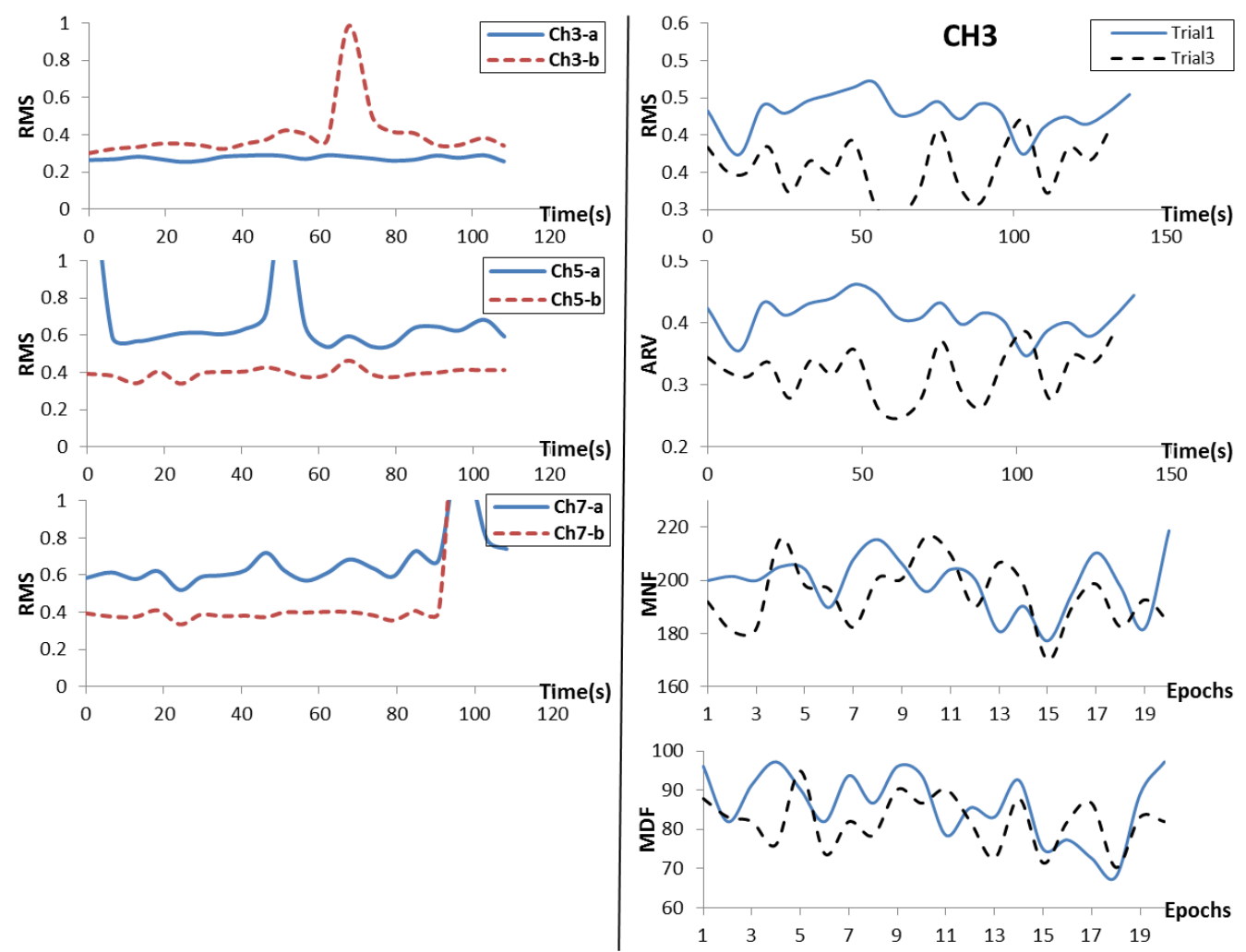

Figure 4- Left column: The normalized RMS factor for pairs of sEMG sensors. Right column: sEMG factors for $\mathrm{CH} 3$ in trial1 and trial3. (Subject 2) 


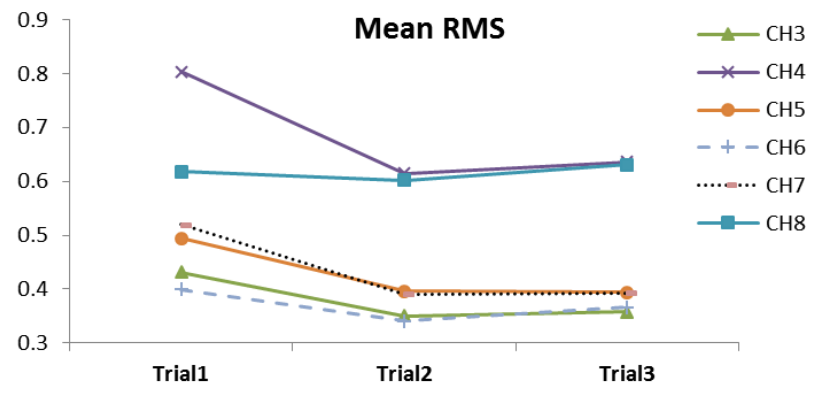

Figure 5-Variation of the mean RMS inter-trials for low back sEMG sensors.

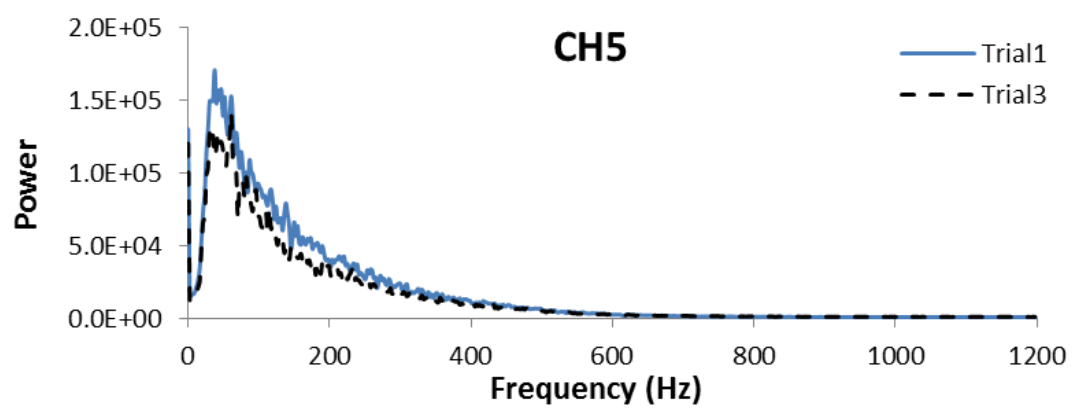

Figure 6-Comparison of Power-Frequency curves obtained from $\mathrm{CH} 5$ in trial1 and trial3

\section{CONCLUSION}

The well-known phenomenon of decreasing RMS and AVR with increasing muscle fatigue was observed in multifidus, longissimus, and iliocostalis muscles (CH3-8). The MDF and MNF parameters did not present a significant relationship between the sEMG and the development of muscle fatigue, as shown in Figure 4. However, the power-frequency curve exhibited the sign of muscle fatigue development, where the curve shifted to the lower frequencies in trial3 compared to the trial1. The sEMG factors did not altered significantly intra-trial, implying that the load or number of repetition were not enough to generate fatigue in a trial. However, the inter-trial variation of RMS and AVR were substantial. As conclusion, only RMS and AVR parameters, along with the power-frequency curve were found potential candidates to indicate the development of muscle fatigue during the investigated isotonic task. In fact, the sEMG data was affected by the underlying muscles in deeper layers in the vicinity of the sensor. Therefore, sEMG data could hardly be correlated to a specific muscle activity in the low back area, where several muscles are crossing each other at a certain point. However, some of the sEMG factors could successfully present the activity of superficial muscle bundles, such as multifidus, longissimus, and iliocostalis.

The integrated limitations of sEMG technique restrain its application in muscle fatigue identification for the entire body. However, it is a functional technique to non-invasively measure the in-vivo muscle activity while performing the workstation task activity. The next step of this study is to develop a human body model, by which the distribution of forces and moments in the body segments could be predicted for variety of task maneuver. 


\section{ACKNOWLEDGEMENTS}

The authors would like to give special thanks to Dr. M. Al-Hussein and Mrs. H. Karimipour for their helpful contribution towards completing the experiments and data analysis.

\section{REFERENCES}

Atlas, S. J., \& Deyo, R. A. (2001). Evaluating and managing acute low back pain in the primary care setting. Journal of General Internal Medicine, 16(2), 120-31. Retrieved from http://www.pubmedcentral.nih.gov/articlerender.fcgi?artid=1495170\&tool=pmcentrez\&ren dertype $=$ abstract

Bruno, A. G., Bouxsein, M. L., \& Anderson, D. E. (2015). Development and validation of a musculoskeletal model of the fully articulated thoracolumbar spine and rib cage. Journal of Biomechanical Engineering. doi:10.1115/1.4030408

Cao, H., El Hajj Dib, I., Antoni, J., \& Marque, C. (2007). Analysis of muscular fatigue during cyclic dynamic movement. Conference Proceedings : ... Annual International Conference of the IEEE Engineering in Medicine and Biology Society. IEEE Engineering in Medicine and Biology Society. Annual Conference, 2007, 1880-3. doi:10.1109/IEMBS.2007.4352682

Georgakis, A., Stergioulas, L. K., \& Giakas, G. (2003). Fatigue analysis of the surface EMG signal in isometric constant force contractions using the averaged instantaneous frequency. IEEE Transactions on Bio-Medical Engineering, 50(2), 262-5. doi:10.1109/TBME.2002.807641

Hildebrandt, V. H. (1995). Back pain in the working population: prevalence rates in Dutch trades and professions. Ergonomics, 38(6), 1283-98. doi:10.1080/00140139508925188

Kim, G., Ahad, M., Ferdjallah, M., \& Harris, G. (2007). Correlation of muscle fatigue indices between intramuscular and surface EMG signals. In Proceedings 2007 IEEE SoutheastCon (pp. 378-382). IEEE. doi:10.1109/SECON.2007.342928

Kranz, H., Williams, A. M., Cassell, J., Caddy, D. J., \& Silberstein, R. B. (1983). Factors determining the frequency content of the electromyogram. Journal of Applied Physiology: Respiratory, Environmental and Exercise Physiology, 55(2), 392-9. Retrieved from http://www.ncbi.nlm.nih.gov/pubmed/6311777

Lindström, L., Kadefors, R., \& Petersén, I. (1977). An electromyographic index for localized muscle fatigue. Journal of Applied Physiology: Respiratory, Environmental and Exercise Physiology, 43(4), 750-4. Retrieved from http://www.ncbi.nlm.nih.gov/pubmed/583632

Mananas, M. A., Rojas, M., Mandrile, F., \& Chaler, J. (2005). Evaluation of muscle activity and fatigue in extensor forearm muscles during isometric contractions. Conference Proceedings : ... Annual International Conference of the IEEE Engineering in Medicine and Biology Society. IEEE Engineering in Medicine and Biology Society. Annual Conference, 6, 5824-7. doi:10.1109/IEMBS.2005.1615813 
Mannion, A. F., \& Dolan, P. (1994). Electromyographic median frequency changes during isometric contraction of the back extensors to fatigue. Spine, 19(11), 1223-9. Retrieved from http://www.ncbi.nlm.nih.gov/pubmed/8073313

Marshall, P. W. M., Lovell, R., Knox, M. F., Brennan, S. L., \& Siegler, J. C. (2015). Hamstring fatigue and muscle activation changes during six sets of Nordic hamstring exercise in amateur soccer players. Journal of Strength and Conditioning Research, 1. doi:10.1519/JSC.0000000000000966

Perry, J., Easterday, C. S., \& Antonelli, D. J. (1981). Surface versus intramuscular electrodes for electromyography of superficial and deep muscles. Physical Therapy, 61(1), 7-15. Retrieved from http://www.ncbi.nlm.nih.gov/pubmed/7454803

Roy, S. H., De Luca, C. J., \& Casavant, D. A. (1989). Lumbar muscle fatigue and chronic lower back pain. Spine, 14(9), 992-1001. Retrieved from http://www.ncbi.nlm.nih.gov/pubmed/2528828

Sarmiento, J. F., Benevides, A. B., Moreira, M. H., Elias, A., Bastos, T. F., Silva, I. V, \& Pelegrina, C. C. (2011). Comparative muscle study fatigue with sEMG signals during the isotonic and isometric tasks for diagnostics purposes. Conference Proceedings : ... Annual International Conference of the IEEE Engineering in Medicine and Biology Society. IEEE Engineering in Medicine and Biology Society. Annual Conference, 2011, 7163-6. doi:10.1109/IEMBS.2011.6091810

Segizbaeva, M. O., \& Aleksandrova, N. P. [Inspiratory muscle resistance to fatigue during exercise and simulated airway obstruction]. Fiziologiia Cheloveka, 40(6), 114-22. Retrieved from http://www.ncbi.nlm.nih.gov/pubmed/25711114

Sparto, P. J., Parnianpour, M., Reinsel, T. E., \& Simon, S. (1997). Spectral and temporal responses of trunk extensor electromyography to an isometric endurance test. Spine, 22(4), 418-25; discussion 425-6. Retrieved from http://www.ncbi.nlm.nih.gov/pubmed/9055371

Vera-Garcia, F. J., Moreside, J. M., \& McGill, S. M. (2010). MVC techniques to normalize trunk muscle EMG in healthy women. Journal of Electromyography and Kinesiology: Official Journal of the International Society of Electrophysiological Kinesiology, 20(1), 10-6. doi:10.1016/j.jelekin.2009.03.010

Vøllestad, N. K. (1997). Measurement of human muscle fatigue. Journal of Neuroscience Methods, 74(2), 219-27. Retrieved from http://www.ncbi.nlm.nih.gov/pubmed/9219890

Woźniak, K., Lipski, M., Lichota, D., \& Szyszka-Sommerfeld, L. (2015). Muscle fatigue in the temporal and masseter muscles in patients with temporomandibular dysfunction. BioMed Research International, 2015, 269734. doi:10.1155/2015/269734 